\title{
Treatment of trichiasis with a lid cryoprobe
}

\author{
R L C JOHNSON AND J R O COLLIN
}

From Moorfields Eye Hospital, City Road, London EC1V 2PD

SUMMARY A series of 112 eyelids with symptomatic aberrant eyelashes were treated by cryotherapy using a specially designed large-surfaced high-flow nitrous oxide probe. Either thermocouple control to $-20^{\circ} \mathrm{C}$ or timing of the application to 20 seconds (lower lid) or 25 seconds (upper lid) was used to monitor the freeze. Both techniques obtained a high cure rate and a low incidence of complications.

Misdirected and aberrant eyelashes causing symptomatic ocular discomfort are most frequently described as trichiasis, but the irritating hairs on the lid margin skin after eyelid reconstruction and the second row of lashes in congenital distichiasis also cause similar problems. Any of these types of abnormal and irritating hairs can be treated with cryotherapy to destroy their follicles.

The management of trichiasis has variously included radiotherapy, ${ }^{1}$ surgery, ${ }^{2}$ electrolysis ${ }^{3}{ }^{3}$ and cryotherapy. ${ }^{-14}$ Radiotherapy and surgery, although efficacious, can result in excessive structural and functional complications and have been generally discarded except in pigmented patients where surgery can play a useful role. Electrolysis can satisfactorily destroy a hair follicle but has to be discretely, carefully, and tediously applied to each separate follicle, and although of doubtless benefit for the treatment of a few and scattered eyelashes there is a significant risk of excessive scarring of the lids if a large area or number of lashes require treatment. ${ }^{3}$ Cryotherapy is well established as a method of eradicating hair follicles, but the standard retinal cryoprobe using either carbon dioxide or nitrous oxide as the freezing agent has an unacceptable incidence of failures $(50 \%$ or greater $),{ }^{59}$ and liquid nitrogen, though effective, can be technically difficult to use. ${ }^{712}$ We report on our experiences with a nitrous oxide probe with a high flow rate of gas and a modified tip with a large surface area specially designed for the treatment of trichiasis of the lid margins. We believe it can provide a safe and effective instrument in all surgeons' hands.

Correspondence to J R O Collin, FRCS.

\section{Material and methods}

The standard technique for abolishing eyelashes by the Collin cryoprobe was performed under clean but not sterile conditions. The skin was prepared for injection with an alcohol swab, and amethocaine $1 \%$ drops were instilled into the conjunctival sac. $2 \%$ Lignocaine with adrenaline $(1: 100000)$ was locally infiltrated beneath skin and conjunctiva to obtain anaesthesia and vasoconstriction. In those patients in whom the temperature was monitored a microthermocouple was placed at the base of the hair follicles to be treated, and an insulating plate (a plastic coffee spoon) was used to protect the globe. The probe was applied from either the conjunctival or skin surface depending on the position of the targeted lashes and a thermocouple temperature reading of $-20^{\circ} \mathrm{C}$ at the lash follicle was obtained. Later in the series the thermocouple was not used, as it had been observed that the time required to obtain the desired temperature was consistently 20 seconds in the lower lid and 25 seconds in the upper lid (Table 1). After the freeze was completed the probe was removed and the tissues allowed to thaw spontaneously until a positive temperature was recorded or until no ice formation was visible if the thermocouple was not used. No heat (e.g., irrigation) was applied to hasten the thaw. The cycle was then repeated. If multiple areas of application along the eyelid were required, an adequate freeze was ensured in the junctional areas by a slight but accurate overlap of the application sites. At the end of the freeze the offending lashes were epilated. A topical antibiotic ointment was applied and the eye firmly padded. The patients were instructed that the eyelid might be painful, usually only for a few hours during the first 12 hours, 
Table 1 Relationship of recurrences to monitoring technique and causes of trichiasis

\begin{tabular}{|c|c|c|c|}
\hline & Patients & Lids & Recurrences (time) \\
\hline \multicolumn{4}{|l|}{ A. Monitoring Technique } \\
\hline Thermocouple & 48 & 89 & 16 lids (14 months) \\
\hline Timing & 14 & 23 & 2 lids ( $7 \cdot 4$ months) \\
\hline \multicolumn{4}{|l|}{ B. Diagnosis } \\
\hline \multicolumn{4}{|l|}{ Idiopathic trichiasis (chronic blepharitis, glaucoma therapy, } \\
\hline Herpes zoster ophthalmicus & 2 & 3 & Nil \\
\hline Congenital distichiasis & 7 & 24 & 30 lashes/10 lids ( 10 months) \\
\hline Fine skin hairs after lid reconstruction & 4 & 5 & Nil \\
\hline $\begin{array}{l}\text { Cicatrising conjunctival disease (Stevens-Johnson syndrome, } \\
\text { mucous membrane pemphigoid, alkaline burn, radiation) }\end{array}$ & 10 & 21 & $\begin{array}{l}1 \text { lash } / 1 \text { lid ( } 7 \text { months). } \\
2 \text { postradiotherapy lids failed }\end{array}$ \\
\hline Others (Rothmund syndrome, Romberg's hemifacial & & & \\
\hline atrophy, 7 th nerve palsy) & 3 & 5 & Nil \\
\hline Total & 62 & 112 & 18 lids \\
\hline
\end{tabular}

and were instructed to take minor analgesics as required. They were warned to expect some swelling and redness which would persist for 5-10 days. The pad was removed at home after 36 to 48 hours, and topical antibiotic drops and ointment were used until the swelling settled. They were seen after 10 days and again after six weeks. Any residual lashes were epilated at these times-the dead follicles releasing their lashes without discomfort or effort.

\section{Results}

Sixty-two patients, a total of 112 eyelids, were treated with the cryoprobe and included in this study. Forty-eight patients-89 eyelids-were monitored with thermocouple control, and 14 patients-23 eyelids-were monitored by timing to 25 seconds (upper lid) or 20 seconds (lower lid). The patients were seen in the clinic and the presence or absence and number of lashes was noted. A total of 23 patients ( 32 eyelids) were unable to attend the clinic after the minimum six-month follow-up time but replied to a questionnaire and were included in the study. They were deemed to be clear of lashes only if no ocular irritation persisted. If any symptoms were present, they were seen in the clinic before inclusion in this review. The follow-up ranged from a minimum of six months to 36 months, and the average for all eyelids was 14 months. The average for lids monitored by timing rather than thermocouple control was $7 \cdot 4$ months. The primary overall success rate for abolition of aberrant lashes was $84 \%(94: 112)$, and in the 23 eyelids under timing control only two eyelashes (one lash in different lids in different patients) recurred, and thus this therapy provided a $91 \%$ cure rate.

The diagnostic subgroups are given in Table 1. Of the 112 lids involved in the study four had been treatment failures from previous cryotherapy using the retinal cryoprobe. Table 1 also shows in which groups the recurrences occurred. In the group monitored with timing only, the recurrence rate was $9 \%$, which represented one recurrent eyelash on two separate eyelids. The overall recurrence rate was $16 \%$. Six patients (with a total of 13 treated eyelidsi.e., $11 \%$ of all lids) had an average of two recurrent lashes per patient, and these were amenable to electrolysis. The only complete failure was in a patient who had previously undergone radiotherapy for a sphenoidal ridge meningioma and later had cryotherapy to the ipsilateral scarred eyelids. She underwent a second cryotherapy treatment, which completely succeeded in ablating all lashes without loss of lid tissue. There were four patients who developed aberrant eyelashes in areas that had not previously been treated. These were not considered to be either recurrences or complications. Whether they were cases of cryotherapy stimulating the adjacent eyelid to produce trichiasis type lashes, progressive disease, or an inadequate extent of initial cryoprobe application remains uncertain. Of the seven patients and 24 eyelids with congenital distichiasis who were treated with cryotherapy three patients had recurrences in a total of 10 eyelids. One patient needed electrolysis for two lashes and two brothers needed more extensive electrolysis for 2-6 abnormal lashes persisting on each eyelid. Significantly, none of the normal lashes which it was intended to preserve were destroyed by the cryotherapy.

In the entire series only four patients had lid swelling judged as marked. The worst case developed a conjunctival pseudomembrane and hemifacial oedema which spread into the neck following simultaneous treatment of two eyelids on the same side. The four patients with excessive swelling were not related to any particular diagnostic group: three 
of them had non-specific trichiasis and one had ocular pemphigoid. All patients had been warned of depigmentation. In this series the only non-white Caucasian patients were two Orientals and neither had significant cosmetic depigmentation. Of the 60 white Caucasian patients only one complained of depigmentation. Interestingly, one patient showed an increase in pigment in the area treated. This was best seen with the slit-lamp as tiny intraepithelial freckles. One patient with chronic blepharitis and trichiasis developed a small lid notch, but in the other cases, including those with reconstructed eyelids, there was no loss of tissue or necrosis, though some slight lid thinning could be observed if extensive areas of the eyelid had been treated.

\section{Discussion}

The biological effect of freezing has been documented by earlier investigators. ${ }^{10}$ is To review very briefly the features of cryobiology pertinent to the treatment of trichiasis, it has been established that a temperature of approximately $-20^{\circ} \mathrm{C}$ is necessary to destroy hair follicles and $-30^{\circ} \mathrm{C}$ to destroy tumour cells, but skin melanocytes are susceptible to higher temperatures of about $-10^{\circ} \mathrm{C} .^{10} 12$ is These temperatures are the temperature present in the lesion or follicle, not on the skin or probe surface. The cryosurgical result is dependent on a rapid freeze and a slow thaw, and the actual effect is greatly improved if a double cycle freeze-thaw, freeze-thaw, is used..$^{10}$ Local vasoconstriction, by means of a local anaesthetic that contains adrenaline, improves the efficiency of the treatment by increasing the depth of the freeze and prolonging the thaw stage.

The treatment of trichiasis with freezing has been successfully performed with liquid nitrogen or nitrous oxide probes, and the thermocouple has been essential. ${ }^{72}{ }^{13}$ Liquid nitrogen, with the very low boiling point of $-195^{\circ} \mathrm{C}$ is a very effective cryo agent, but has the disadvantages of the liquid spreading and running during its application, difficulty of storage, and logistics of acquisition and maintenance. ${ }^{12}{ }^{13} \mathrm{It}$ is, however, effective. ${ }^{7}$ Nitrous oxide, although not as efficient a heat sink as liquid nitrogen, has a lower boiling point than carbon dioxide $\left(-90^{\circ} \mathrm{C}\right.$ versus $-30^{\circ} \mathrm{C}$ ) and thus is more effective than that gas. ${ }^{15}$ Nitrous oxide probes, in the form of cryoprobes, have been limited by low flow systems and small surface areas which limit the efficiency of obtaining an adequate freeze. In the treatment of trichiasis a $50 \%$ or higher failure rate has been reported with them. ${ }^{59}$ To utilise the convenience of the nitrous oxide probe and to improve on the inadequate freezing potential of retinal cryoprobes, a high flow and large surfaced $(10 \times 4 \times 3 \mathrm{~mm})$ probe with two differently orientated surfaces for ease of application has been especially designed for use on eyelids. With thermocouple monitoring to $-20^{\circ} \mathrm{C} 89$ eyelids were treated, with a high success rate. The duration of the application necessary was observed to be consistently 20 seconds for the lower lid and 25 seconds for the upper lid. In the last 23 eyelids the cryosurgical application was monitored by timing for these periods without using a thermocouple. Satisfactory results after a minimum six-month follow-up were obtained.

The overall success rate for the abolition of aberrant lashes was $84 \%$ of eyelids (94/112) in $84 \%$ of patients (52/62). Since only one lash recurred in six lids and four lashes recurred in two lids, all but 10 eyelids were amenable to minor electrolysis to achieve a cure rate of $91 \%$. Of the remaining 10 eyelids, the two postradiotherapy eyelids were cleared of eyelashes with a second cryotherapy treatment, and the other recurrences were mainly in the distichiasis group and required more extensive electrolysis. The diagnostic group with the highest failure rate was these congenital distichiasis patients, of whom three out of seven (nine out of 23 eyelids) had recurrences. However, since these occurred where the stated goal was to spare normal eyelashes, it is likely that the cryotherapy was not applied adequately. The other diagnostic groups, including subgroups for lid reconstruction and cicatrising conjunctival disease, had no particular failure or complication tendency. Our overall success rate of $84 \%$ is about the same as that reported by Wood and Anderson $(90 \%)^{13}$ and Sullivan (88\%) 11 using nitrous oxide. It was much higher than when a retinal probe was used. ${ }^{59}$ If the patients with congenital distichiasis are omitted, our results are similar to those reported by Fraunfelder and Petursson using liquid nitrogen $(90 \%+) .^{7}$

Wood and Anderson ${ }^{13}$ report an incidence of complications of $18 \%$ when using a nitrous oxide probe on the eyelids in the treatment of trichiasis. We suffered a $10 \%$ eyelid complication rate. Four patients suffered marked postoperative oedema, and one of these had a pseudomembrane. These patients had been suffering from idiopathic trichiasis (three patients) or ocular pemphigoid (one patient). All the complications in our patients resolved except for one patient with trichiasis who was left with a lid notch. One patient had cosmetically evident depigmentation, and one had microscopic evidence of hyperpigmentation which was insignificant to the unaided eye. Unlike Wood and Anderson's series ${ }^{13}$ the diagnostic subgroups of cicatrising conjunctival disease and reconstructed lids did not have excessive complications. No corneal disease, acceleration of cicatrisation, or activation of herpes zoster occurred. Thus we 
have not found cryotherapy to be contraindicated in these patients.

We conclude that by using the probe and technique described it is possible consistently to obtain a very high rate of eradication of hair follicles with a low risk of complications and without necessarily using a thermocouple.

R L C Johnson was sponsored by the E A Baker Foundation in Ontario, Canada.

\section{References}

1 McDonald JE, Wilson FM. Ocular therapy with beta particles. Trans Am Acad Ophthalmol Otolaryngol 1959; 63: 468-85.

2 Clorfeine GS, Kielor RA. Lash excision in trichiasis. Ann Ophthalmol 1977; 9: 525-8.

3 Soll DB. Management of complications in ophthalmic plastic surgery. Birmingham, Alabama: Aesculapius, 1976.

4 Sullivan JH, Beard C, Bullock JD. Cryosurgery for treatment of trichiasis. Am J Ophthalmol 1977; 82: 117-21.
5 Collin JRO, Coster DJ, Sullivan JH. Cryosurgery for trichiasis. Trans Ophthalmol Soc UK 1978; 98: 81-3.

6 Fraunfelder FT, Farris HE, Wallace TR, et al. The role of cryosurgery in external ocular and periocular disease. Trans Am Acad Ophthalmol Otolaryngol 1977; 83: 713-24.

7 Fraunfelder FT, Petursson GJ. The use of liquid nitrogen cryospray for treatment of trichiasis. Ophthalmic Surg 1979; 10: 42-6.

8 Hecht SD. Cryotherapy of trichiasis with use of the retinal cryoprobe. Ann Ophthalmol 1977; 90: 1501-3.

9 Majekodunmi S. Cryosurgery in treatment of trichiasis. $\mathrm{Br} \mathrm{J}$ Ophthalmol 1982; 66: 337-9.

10 Soll DB, Harrison SE. Basic concepts and an overview of cryosurgery in ophthalmic plastic surgery. Ophthalmic Surg 1979; 10: $31-6$.

11 Sullivan JH. The use of cryotherapy for trichiasis. Trans Am Acad Ophthalmol Otolaryngol 1977; 83: 708-12.

12 Sullivan JH. Cryosurgery in ophthalmic practice. Ophthalmic Surg 1979; 10: 37-41.

13 Wood JR, Anderson RL. Complications of cryosurgery. Arch Ophthalmol 1981; 99: 460-3.

14 Wingfield DL, Fraunfelder FT. Possible complications secondary to cryotherapy. Ophthalmic Surg 1979; 10: 47-55.

15 Wilkes TDI, Fraunfelder FT. Principles of cryosurgery. Ophthalmic Surg 1979; 10: 21-30. 